Clara Pérez-Barrios*, Estela Sánchez-Herrero, Natalia Garcia-Simón, Miguel Barquín, Mariola Blanco Clemente, Mariano Provencio and Atocha Romero

\title{
ctDNA from body fluids is an adequate source for EGFR biomarker testing in advanced lung adenocarcinoma
}

https://doi.org/10.1515/cclm-2020-1465

Received October 2, 2020; accepted March 1, 2021;

published online March 11, 2021

\section{Abstract}

Objectives: Epidermal growth factor receptor (EGFR) biomarker testing using blood-based liquid biopsies remains challenging due to the low concentration of circulating tumor DNA (ctDNA) in certain plasma samples. The aim of this study is to evaluate the usefulness for EGFR biomarker testing of ctDNA from pleural effusions, cerebrospinal fluids, ascites and pericardial effusions obtained during the clinical management of lung adenocarcinoma patients.

Methods: For comparison purposes, 23 paired plasma and body fluid samples were collected from 17 patients with EGFR-positive lung adenocarcinoma. After circulating free DNA (cfDNA) isolation, samples were evaluated for the initial EGFR-sensitizing mutation and the p.T790M resistance mutation by array-based digital PCR (dPCR).

Results: Body fluids had more cfDNA than plasma samples (1.90 vs. $0.36 \mathrm{ng} / \mu \mathrm{L} ; \mathrm{p}=0.0130)$, and more samples tested positive for EGFR mutations (21 vs. 16 samples), with a total of 28 vs. 22 variants detected. Furthermore, mutant

*Corresponding author: Clara Pérez Barrios, PharmD, PhD, Laboratory Medicine Department, Hospital Universitario Puerta de Hierro-Majadahonda, C/Manuel de Falla 1, Majadahonda, Madrid 28222, Spain, E-mail: clara_pb@hotmail.com

Estela Sánchez-Herrero and Miguel Barquín, Molecular Oncology Laboratory, Biomedical Sciences Research Institute, Hospital Universitario Puerta de Hierro-Majadahonda, Madrid, Spain. https:// orcid.org/0000-0002-2805-0312 (E. Sánchez-Herrero) Natalia Garcia-Simón, Laboratory Medicine Department, Hospital Universitario Puerta de Hierro-Majadahonda, Madrid, Spain Mariola Blanco Clemente and Mariano Provencio, Medical Oncology Department, Hospital Universitario Puerta de Hierro-Majadahonda, Madrid, Spain

Atocha Romero, Molecular Oncology Laboratory, Biomedical Sciences Research Institute, Hospital Universitario Puerta de Hierro-

Majadahonda, Madrid, Spain; and Medical Oncology Department, Hospital Universitario Puerta de Hierro-Majadahonda, Madrid, Spain. https://orcid.org/0000-0002-1634-7397 allele frequencies (MAFs) observed in body fluids were significantly higher than those assessed in the paired plasma samples for EGFR-sensitizing mutations (median MAFs $=15.8$ vs. $0.8 \% ; \mathrm{p}=0.0004)$ as well as for the $\mathrm{p} . \mathrm{T} 790 \mathrm{M}$ resistance mutation (median MAFs $=8.69$ vs. $0.16 \%$; $\mathrm{p}=0.0390$ ). Importantly, two patients who had progressed on first-generation $E G F R$-tyrosine kinase inhibitors with a dubious result for p.T790M plasma (MAFs $=0.11 \%$ ) had an indisputably positive result in their respective body fluid samples (MAFs $=10.25$ and $9.66 \%$ ).

Conclusions: ctDNA derived from body fluids is an informative source for EGFR biomarker testing, with greater sensitivity than plasma samples.

Keywords: biomarker testing; body fluids; ctDNA; EGFR; liquid biopsy; lung adenocarcinoma.

\section{Introduction}

In the last decade, biomarker testing has become very important in the management of advanced lung adenocarcinoma because it allows for the selection of patients who can benefit from targeted therapies. For guiding treatment decisions, clinical guidelines strongly recommend testing for markers of a variety of genes, such as $E G F R, A L K, R O S 1$, and BRAF, as well evaluating PDL-1 expression [1].

EGFR mutational status is typically evaluated in a tissue biopsy taken at the time of diagnosis, which enables response to EGFR-tyrosine kinase inhibitors (TKIs) to be predicted [2]. Additionally, some acquired mutations might appear under TKI treatment, of which p.T790M is the most frequent resistance mutation. However, the availability of tumor tissue obtained from biopsies in lung adenocarcinoma patients is sometimes low, which could limit the analysis of biomarkers. In addition, the analysis of liquid biopsies is significantly faster and enables the determination of the molecular profiles of the tumor throughout the course of the disease and the detection of clinically relevant alterations caused by the treatments. In this context, circulating tumor DNA (ctDNA), which is the fraction of 
circulating free DNA (cfDNA) that comes from cancerous cells and tumors, has been shown to be an adequate source for the identification and quantification of molecular alterations of the EGFR gene that can be used to monitor the course of the disease and detect relapse during targeted therapies $[3,4]$. In addition, the analysis of ctDNA from plasma has the advantage that sample collection is straightforward, having been integrated into the clinical routine of many laboratories. Nonetheless, the amount of ctDNA in plasma is affected by many factors, such as tumor size or location [5, 6], which can limit the sensitivity of this approach in biomarker testing.

Body fluids, such as pleural effusion (PE), cerebrospinal fluid (CSF), ascites (ASC) and pericardial effusion (PC), are occasionally obtained from lung adenocarcinoma relapses in the course of clinical management. Although its collection is more invasive than that of blood, supernatant from other body fluids contains more ctDNA than its plasma counterparts $[7,8]$, making them more sensitive for tumor typing. Furthermore, the proximity of certain body fluids to the tumor site can be useful for obtaining molecular information from localized metastases, such as those in the brain, in which the amount of DNA released into the blood is usually very low [9].

In the present study, we evaluated the usefulness of ctDNA obtained from body fluids for EGFR molecular testing. To this end, 23 paired samples of plasma and body fluid were collected from 17 patients with EGFR-positive lung adenocarcinoma. After cfDNA isolation, the original sensitizing EGFR mutation and the p.T790M resistance mutation were assessed in both specimens using digital PCR (dPCR) for comparison purposes. Finally, we specifically analyzed the p.T790M mutation in body fluids obtained at progression to first- or second-generation TKIs to assess its impact on clinical decision-making.

\section{Materials and methods}

\section{Study population}

This is a non-interventional prospective study in which specimens were collected during routine clinical practice, according to the oncologist's criteria. Specifically, 23 body fluid samples from 17 nonsmall cell lung cancer (NSCLC) patients were collected between November 2015 and March 2020, after patients had provided their signed informed consent. All patients had been diagnosed with advanced lung adenocarcinoma disease (stage IV) and had biopsyproven EGFR-sensitizing mutations using the Cobas ${ }^{\circledR}$ EGFR Mutation Test CE-IVD (Roche Molecular Systems, Pleasanton, CA, USA). Additionally, 23 blood samples were collected from the same cohort of patients, with a maximum of three weeks between body fluid and matched plasma sample collection. Information regarding clinicopathological features and tumor mutation status were obtained from clinical records. The study protocol was approved by the Hospital Puerta de Hierro Ethics Committee (internal code 144/14).

\section{Laboratory procedures}

Body fluids were collected in additive-free containers and blood samples were kept in $8.5 \mathrm{~mL}$ PPT $^{\mathrm{TM}}$ tubes (Becton Dickinson). Immediately upon arrival at the laboratory, both samples were centrifuged at $1,500 \mathrm{~g}$ for $10 \mathrm{~min}$ and the supernatant was separated using Falcon $^{\mathrm{TM}} 15 \mathrm{~mL}$ conical tubes and stored at $-80^{\circ} \mathrm{C}$. On the day the cfDNA was extracted, samples were thawed in the refrigerator at $4^{\circ} \mathrm{C}$ and additional centrifugation was performed at 5,000 $\mathrm{g}$ for $20 \mathrm{~min}$ to ensure removal of cells and debris. The Maxwell ${ }^{\circledR}$ RSC (MR) ccfDNA Plasma Kit (Promega Corporation, Madison, WI, USA) was then used for cfDNA isolation, following the manufacturer's instructions and using the " $1 \mathrm{~mL}$ cell free DNA custom" program. For the final elution, $50 \mu \mathrm{L}$ of the supplied buffer were used. The cfDNA obtained was stored at $-20{ }^{\circ} \mathrm{C}$ until further evaluation.

\section{EGFR mutation quantification}

Samples were thawed at $4{ }^{\circ} \mathrm{C}$ and cfDNA was quantified using the QuantiFluor ${ }^{\circledR}$ dsDNA System Kit in a Quantus Fluorometer (Promega Corporation, Madison, WI, USA). Subsequently, cfDNA was analyzed by array-based PCR QuantStudio ${ }^{\circledR}$ 3D Digital PCR System (Applied Biosystems, South San Francisco, CA, USA) using commercially available predesigned TaqMan ${ }^{\circledR}$ Liquid Biopsy dPCR assays for EGFR mutation detection and quantification. Specifically, five assays were used to detect some of the most frequent exon 19 deletions: EGFR p.E746_A750del (ELREA deletion, NM_005228.3:c.2235_2249del15 and NM_005228.3:c.2236_2250del15), EGFR p.L747_T751del (LREAT deletion, NM_005228.3:c.2239_2253del15), EGFR p.L747_S752del (LREATS deletion, NM_005228.3:c.2239_2256del18), and EGFR p.L747_A750delinsP (LREA >P indel, NM_005228.3:c.2239_2248delinsC), while the EGFR p.L858R and p.T790M (NM_005228.5:c.2573T $>\mathrm{G}$ and NM_005228.5:c.2369C >T, respectively) were analyzed through their specific assays.

For each reaction, $8.55 \mu \mathrm{L}$ of template cfDNA was mixed with $0.45 \mu \mathrm{L}$ of the $40 \times$ specific TaqMan ${ }^{\circledR}$ assay and $9 \mu \mathrm{L}$ of QuantStudio ${ }^{\circledR} 3 \mathrm{D}$ Digital PCR Master Mix v2. After that, $14.5 \mu \mathrm{L}$ of this mixture were loaded onto QuantStudio 3D Digital PCR 20K chips for the purpose of amplification. PCR cycling was carried out under the previously described conditions, and data were analyzed with QuantStudio ${ }^{\circledR}$ 3D Analysis Suite $^{\mathrm{TM}}$ Cloud Software [10]. For each assay, results were reported as the mutant allele frequency (MAF), which is calculated as the ratio of mutant DNA molecules to the sum of mutant and wildtype (wt) DNA molecules. The limit of detection for each assay was calculated following the recommendations of The International Council for Harmonisation of Technical Requirements for Pharmaceuticals for Human Use (ICH) Guidelines [11], which have been published elsewhere [4]. The sensitivity and specificity of the assays, considering tissue genotyping to be the gold standard, have also been reported [12]. According to established custom, samples were considered to be positive when the MAF was greater than or equal to $0.1 \%$ and when there were at least 300 copies/mL of wt DNA. A negative control DNA (wt) and a blank sample (containing no DNA) were included in every run. 


\section{Statistical analysis}

The nonparametric comparison of cfDNA concentration and MAFs in paired samples was evaluated using the Wilcoxon signed-rank test. MAFs were compared nonparametrically with respect to mutation type (del19 vs. p.L858R) and cytology result (positive vs. negative/insufficient) using Mann-Whitney U tests. Finally, Kruskal-Wallis tests were used for comparisons with respect to the time of sample collection (diagnosis, follow-up, or progression). Null hypotheses were rejected if the probability of a type I error was less than 0.05. Statistical analyses were carried out using packages in R 3.6.2.

\section{Results}

\section{Study cohort}

The study population consisted of 17 advanced lung adenocarcinoma patients with biopsy-proven EGFRsensitizing mutations. Specifically, eight of them carried an exon 19 deletion and the other nine bore the p.L858R substitution. A majority of patients were female $(n=10)$, and former or never smokers $(n=15)$. Patient age ranged from 49 to 81 years. Comprehensive clinicopathological characteristics of the study population are summarized in Supplementary Table 1.

From this cohort of patients, a total of 23 body fluid samples were collected according to the oncologist's criteria: 15 PE, five CSF, two PC, and one ASC. Based on the time of collection, four samples were obtained at the time of diagnosis, six during follow-up and 13 at progression (Supplementary Figure 1). Cytological results were positive in 11 of the 16 available pathology reports. Additionally, 23 paired blood samples were collected for comparison.

\section{Cell-free DNA concentration}

Once cfDNA had been isolated, we quantified it and compared the results of the paired samples. Overall, cfDNA concentration was significantly higher in body fluids than in plasma samples $(\mathrm{p}=0.0130)$, with medians of $1.90 \mathrm{ng} / \mu \mathrm{L}$ [interquartile range $(\mathrm{IQR})=0.37-5.13 \mathrm{ng} / \mu \mathrm{L}$ ] and $0.36 \mathrm{ng} /$ $\mu \mathrm{L}[\mathrm{IQR}=0.14-0.76 \mathrm{ng} / \mu \mathrm{L}]$, respectively (Figure $1 \mathrm{~A}$ ). However, quantitation revealed a wide range of cfDNA concentrations in body fluid samples, from 0.04 to
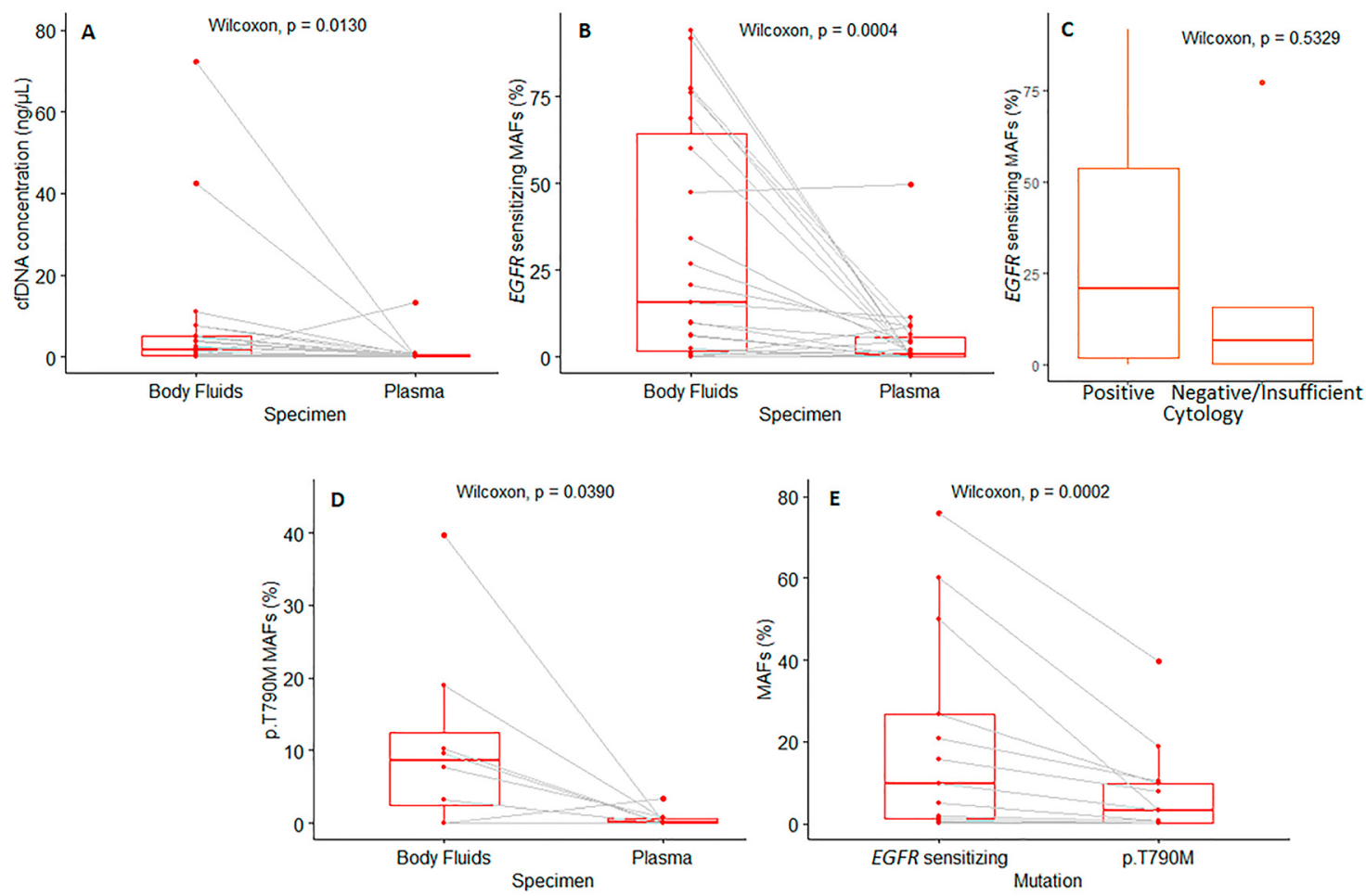

Figure 1: Box-plots representing the distribution of variables analyzed.

(A) cfDNA in paired body fluid vs. plasma samples. (B) EGFR-sensitizing mutation MAFs in paired body fluid vs. plasma samples (C) EGFR-sensitizing mutations in body fluid samples with a positive cytology result vs. samples with a negative result or reported as insufficient for cytological study. (D) p.T790M mutation MAFs in paired body fluid vs. plasma samples. (E) EGFR-sensitizing MAFs vs. p.T790M MAFs in samples presenting both mutations concomitantly. 
$72.20 \mathrm{ng} / \mu \mathrm{L}$. Comparing the location of body fluids, the lowest median cfDNA concentration $(0.18 \mathrm{ng} / \mu \mathrm{L})$ was found in CSF (Supplementary Figure 2). No significant differences in cfDNA concentration with respect to their time of collection were found.

\section{EGFR mutation detection}

Overall, 28 and 22 EGFR variants were identified from body fluid and plasma samples, respectively. Of these mutations, 21 were detected in both samples, while seven were detected only in body fluids and one was only found in plasma samples (Table 1). Given that the EGFR-sensitizing mutations had been biopsy-proven in all patients, but the p.T790M resistance mutation was only acquired by some patients, we decided to analyze the molecular results separately.

\section{EGFR-sensitizing mutations}

Of the 23 body fluids analyzed, we were able to detect 21 EGFR-sensitizing mutations, representing a detection rate of $91.30 \%$. The only two negative results (one exon 19 deletion and one p.L858R substitution) were obtained from cerebrospinal fluids collected at the time of disease progression. Interestingly, four of the five samples reported as negative or insufficient for cytological study were positive by cfDNA. On the other hand, 16 of 23 plasma samples were positive for their known EGFR-sensitizing mutation, representing a detection rate of $69.57 \%$. All the mutations identified in plasma were also positive in the paired bodyfluid samples. These results are summarized in Table 1.

Next, we compared the allele frequencies of the EGFRsensitizing mutations between plasma and other body fluids. Overall, the median MAF observed in body fluids was $15.80 \%$ [IQR $=1.82-64.49 \%$, while it was only $0.80 \%$ $[\mathrm{IQR}=0.00-5.60 \%]$ in plasma samples. This difference was statistically significant according to the Wilcoxon test $(\mathrm{p}=0.0004$; Figure 1B). We then analyzed whether the MAF differences depended on the type of EGFR-sensitizing mutation tested (exon 19 deletion and p.L858R substitution), or on the time of sample collection, but found no significant differences.

Finally, we compared the MAFs obtained in body fluids by cytology. We detected median MAFs of $20.74 \%$ $[\mathrm{IQR}=1.82-53.85 \%]$ in samples with a positive result, and of $6.54 \%$ [IQR $=0.21-15.80 \%$ ] in those reported to be negative or insufficient for cytological study. However, this difference was not statistically significant according to the Mann-Whitney independent samples test (Figure 1C).

\section{p.T790M}

The EGFR p.T790M resistance mutation was detected in seven fluids (including two for which the paired plasma sample was negative), and six plasma samples (including one for which the paired CSF sample was negative).

As shown in Figure 1D, the median MAF of the p.T790M mutation detected in body fluids was significantly higher than that observed in plasma samples $(8.69 \%$ [IQR $=2.41-$ $12.42 \%$ ] vs. $0.16 \%$ [0.10-0.74\%]; $\mathrm{p}=0.0390$ ). Furthermore, in samples in which p.T790M was detected concomitantly with the EGFR-sensitizing mutation ( $\mathrm{n}=13$ ), we found that MAFs of the EGFR-sensitizing mutation were significantly higher than those of the concomitant p.T790M ( $p=0.0002)$ (Figure 1E).

Finally, we specifically analyzed the p.T790M results in 16 paired samples from the eight patients who relapsed while on first- or second-generation TKIs. Overall, we detected four p.T790M resistance mutations in body fluids and five in plasma samples. The only discordant result corresponded to a CSF. Remarkably, although all the p.T790M mutations detected in fluids were also positive in plasma $(n=4)$, the mutation concentration was considerably higher in body fluids than in plasma samples, with respective median MAFs of $14.60 \%$ [IQR $=10.10-24.13 \%$ ] and $0.16 \%[\mathrm{IQR}=0.11-0.33 \%]$. This finding was especially interesting in two cases (Figures 2 and 3), in which the MAF at which the mutation was detected in plasma samples was close to the limit of detection (MAF $=0.1 \%$ ), making it difficult to classify the result as positive or negative. In both examples, paired body fluid samples showed a clearly positive p.T790M result, with MAFs of 10.25 and $9.66 \%$. The patients were treated with osimertinib and their responses lasted 18 and 11 months, respectively.

\section{Discussion}

EGFR biomarker testing from blood-based liquid biopsies has become an essential tool for the clinical management of advanced lung adenocarcinoma patients, providing updated molecular information throughout the course of the disease. However, these studies remain challenging owing to the limited amount of ctDNA present in plasma samples. In the current study, we have thoroughly assessed the clinical usefulness of body fluids for biomarker testing in one of the largest cohorts with paired 


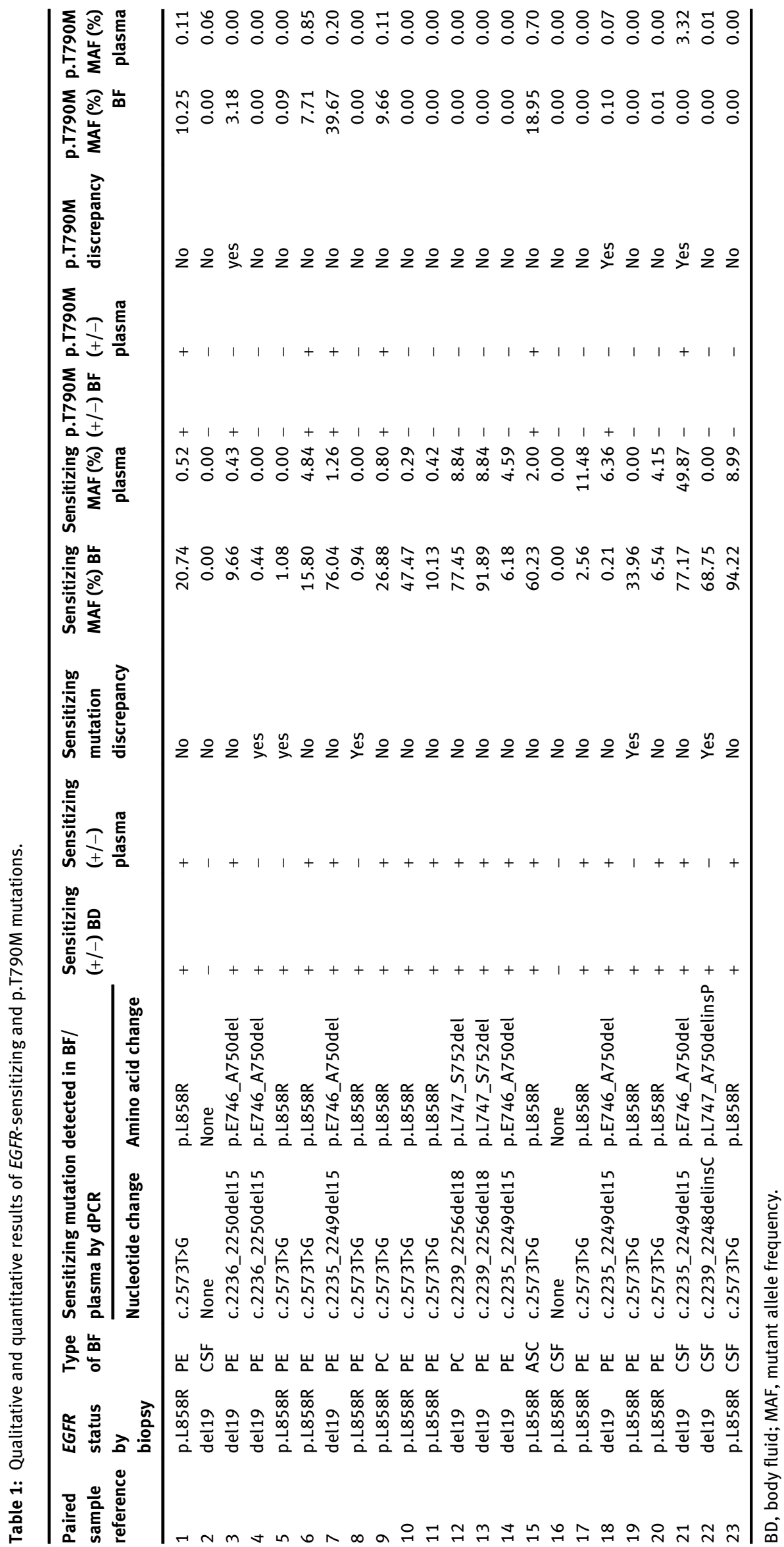



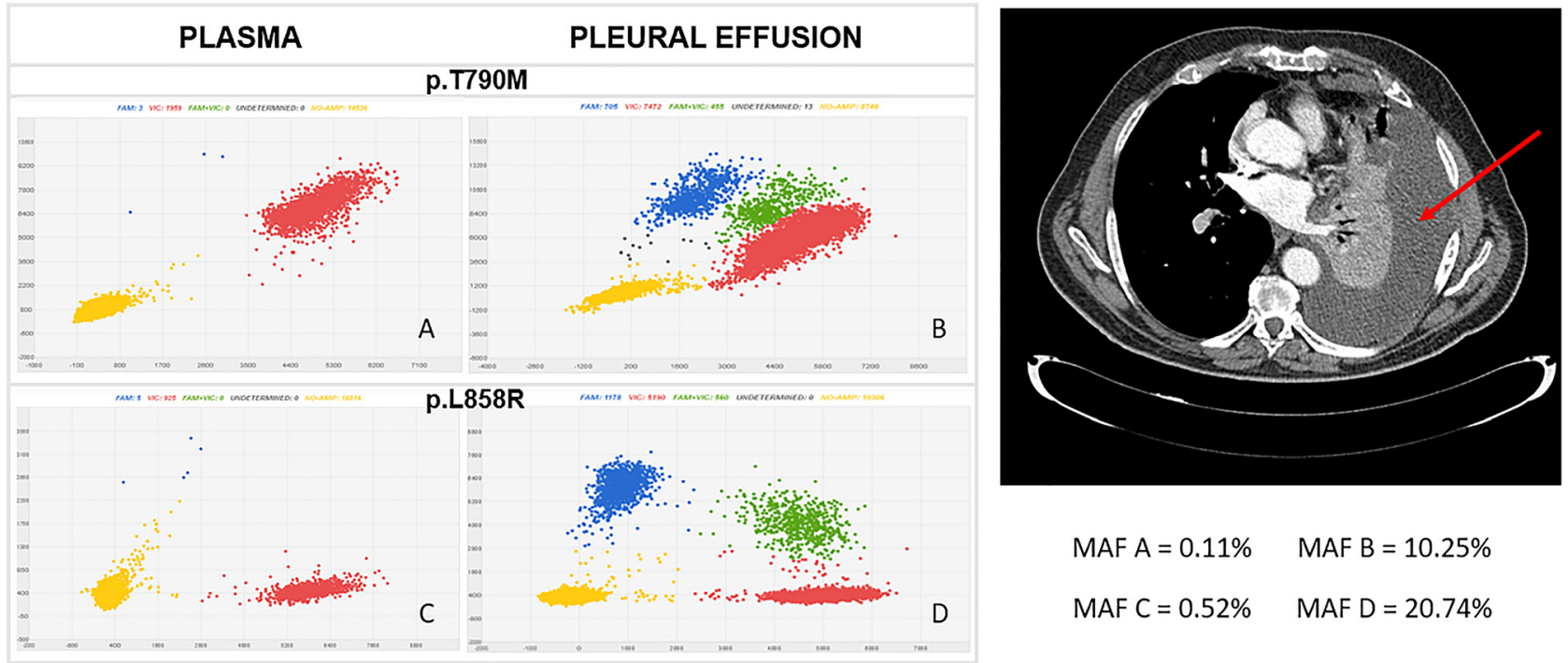

Figure 2: $d P C R$ scatter-plots of p.T790M and p.L858R mutations in paired plasma (A, C) and pleural effusion (B, D) samples, which are evident in the 18-FDG PET-CT scan.

For each assay, the specific mutation is labeled with FAM (blue dots), and the wild type is labeled with VIC (red dots). Green dots represent simultaneous detection of fluorescence in VIC and FAM (more than one copy); yellow dots indicate wells in which there was no amplification. Figure corresponds to pair 1 in Table 1.
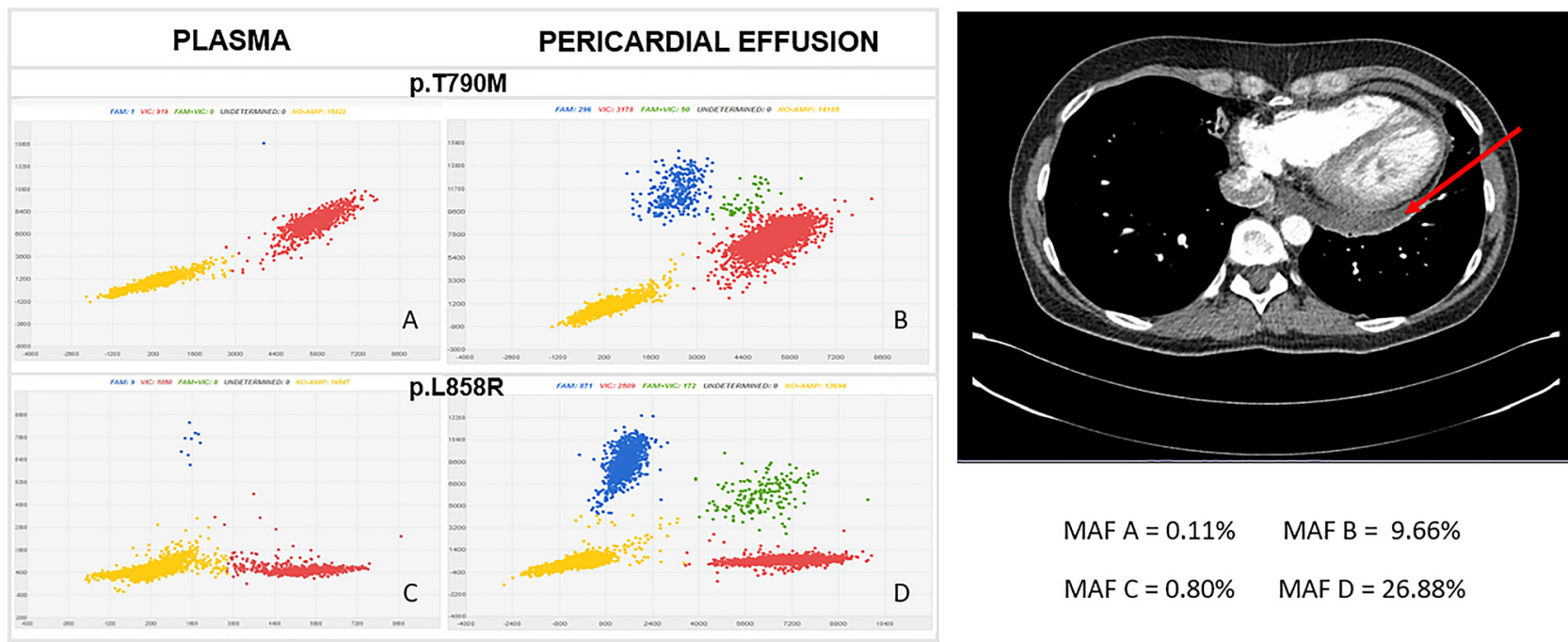

$$
\begin{array}{ll}
\text { MAF A }=0.11 \% & \text { MAF } B=9.66 \% \\
\text { MAF C }=0.80 \% & \text { MAF D }=26.88 \%
\end{array}
$$

Figure 3: dPCR scatter-plots of p.T790M and p.L858R mutations in paired plasma (A, C) and pericardial effusion (B, D) samples, which is evident in the 18-FDG PET-CT scan.

For each assay, the specific mutation is labeled with FAM (blue dots), and the wild type is labeled with VIC (red dots). Green dots represent simultaneous detection of fluorescence in VIC and FAM (more than one copy); yellow dots indicate wells in which there was no amplification. The plasma sample gives a dubious result for p.T790M (A), with a MAF close to the limit of detection. However, the positive result for p.T790M in the pericardial effusion is beyond reasonable doubt, reinforcing the utility of biomarker testing in body fluids. Figure corresponds to pair 9 in Table 1 .

plasma-body fluid reported. The results obtained prove that malignant fluids collected as part of routine clinical practice from advanced lung adenocarcinoma patients, such as PE, CSF, PC and ASC, are suitable alternative samples for molecular profiling, detecting a higher rate of mutations than observed in plasma samples.
First, we found that body fluids were enriched with cfDNA relative to blood. This feature could be especially pertinent for achieving maximum sensitivity when carrying out high-sensitivity next-generation sequencing (NGS), since library preparation frequently requires a minimum quantity of ctDNA input, which can be difficult to 
obtain from plasma [13]. Moreover, this enrichment seems to have an important impact on biomarker testing, conferring greater sensitivity on body fluids than plasma. In this context, we have been able to detect 21 of the total 23 biopsy-proven EGFR-sensitizing mutations using cfDNA from body fluids. This detection rate $(91.30 \%)$ is similar to that previously reported [14] and is higher than the sensitivity of $60-70 \%$ noted in plasma samples $[15,16]$. Interestingly, the two negative body fluids were CSF, which had the lowest cfDNA concentration. However, several authors have demonstrated the utility of cfDNA from CSF for detecting genomic alterations in primary and metastatic brain tumors [17-20], obtaining superior results to those of plasma [9]. The remaining three CSF samples assessed were positive for $E G F R$-sensitizing mutations, with MAFs that ranged from 68.75 to $94.22 \%$. On the other hand, negative results may also be due to an incorrect dPCR assay. In our study, biomarker testing in tissue samples was performed using the Cobas ${ }^{\circledR}$ system, which does not specify which of the EGFR exon 19 deletions is present in a given sample. Since we have used only five specific dPCR assays to detect the most frequent exon 19 deletions, lack of testing for other less common exon 19 deletions could explain the negative result observed in CSF from paired sample reference 2 (Table 1). Unfortunately, there was no tissue available to test the dPCR assays on the solid tumor. Regarding the other negative CSF (paired sample reference 16; Table 1), it belongs to a patient whose tumor harbors the EGFR p.L858R mutation, and therefore the possibility of an incorrect dPCR assay was discarded. Nevertheless, significantly more EGFR-sensitizing mutations were detected in the supernatant of body fluids than in plasma and cytological samples, in which only 16 and six sensitizing mutations, respectively, were reported, in agreement with earlier studies [14, 21].

The sensitivity of body fluids was also demonstrated quantitatively, as described in recent studies [8, 22]. Within this frame of reference, it has been reported [23] that the majority of discordant results between liquid biopsy approaches were found in plasma samples with MAFs $<1 \%$. According to our data, approximately half of the mutations detected in plasma samples (10/22) were below this MAF. Interestingly, this proportion dropped to $4 / 28$ for body fluid samples, which offers a means of solving the specificity problems related to low ctDNA MAFs. Furthermore, we found no significant differences between the MAFs of samples with positive cytology results and those reported as negative or insufficient, in agreement with previous studies [24]. This implies that mutation detection in supernatant may be a useful adjunct for cytological samples with negative or indeterminate diagnoses.
Regarding the results with p.T790M, the frequency and abundance of the mutations detected were again significantly higher in body fluids than in plasma samples. As shown in Figures 2 and 3, this feature is especially useful for discriminating between a positive and a negative result in cases where the patient is relapsing to first- or secondgeneration TKIs, and in which p.T790M is present at a very low concentration in plasma. Finally, we obtained a positive p.T790M result in plasma $(\mathrm{MAF}=3.32 \%)$ that was negative in CSF, which can be explained by the heterogeneous spatial distribution of the p.T790M mutation, as other studies have suggested [25].

The study has some limitations, the most important of which arises from the dPCR approach itself, whereby each mutation must be tested individually, making the study especially laborious when large numbers of them have to be tested. For this reason, and because more than 30 subtypes [26] of mutations are known, an exhaustive study of exon 19 deletions was not possible. Nevertheless, the detection rate for $E G F R$-sensitizing mutations in the supernatant of body fluids was notably high, exceeding $90 \%$. On the other hand, although this study involved one of the largest cohorts with paired plasmabody fluid samples reported $[8,27]$, the fact that obtaining body fluids is more invasive than obtaining plasma limited the number of samples we were able to include ( $\mathrm{n}=23)$.

However, these limitations did not prevent us from demonstrating that body fluids are extremely useful for biomarker testing, leading us to recommend they should form part of molecular analyses whenever possible. Furthermore, the use of alternative high-throughput approaches, such as NGS, may permit extended molecular profiling when tissue samples are exhausted or when a tumor sample is insufficient, of low quality or below the limit of detection for NGS. In this context, the analysis of ctDNA from body fluids by NGS at the time of EGFR-TKI progression can be useful for identifying acquired resistance mutations and planning subsequent treatments. It is worth noting that a wide range of resistance mechanisms are known to occur upon osimertinib failure, such as acquired KRAS mutations and other targetable gene fusions [28]. Likewise, several resistance mutations at the ALK locus are known, although not all of them confer resistance to all $A L K$-TKIs, highlighting the importance of genotyping following disease progression in patients receiving targeted therapies [29].

In summary, ctDNA obtained from body fluids is an adequate source for EGFR biomarker testing in advanced lung adenocarcinoma patients and is more sensitive than plasma samples. 
Acknowledgments: We would like to thank the patients for their participation in the study.

Research funding: $M B$ is supported by an i-PFIS predoctoral fellowship (Grant Number IFI18/00051) from ISCIII. ES is funded by the Consejería de Ciencia, Universidades e Innovación de la Comunidad de Madrid (Doctorados Industriales de la Comunidad de Madrid IND2019/BMD-17258), Spain.

Author contributions: All authors have accepted responsibility for the entire content of this manuscript and have approved its submission.

Competing interests: Authors state no conflict of interest. Informed consent: Informed consent was obtained from all individuals included in this study.

Ethical approval: Our research involving human subjects complied with all the relevant national regulations and institutional policies, and was carried out in accordance with the tenets of the Helsinki Declaration (as revised in 2013), and was approved by the Hospital Puerta de Hierro Ethics Committee (internal code 144/14).

\section{References}

1. Lung cancer metastatic. Available from: NCCN.org/patients NCCN guidelines for patients NON-small cell lung cancer.

2. Gazdar AF. Activating and resistance mutations of EGFR in nonsmall-cell lung cancer: role in clinical response to EGFR tyrosine kinase inhibitors. Oncogene 2009;28:S24-31.

3. Provencio $M$, Torrente M, Calvo V, Gutiérrez L, Pérez-Callejo D, Pérez-Barrios C, et al. Dynamic circulating tumor DNA quantification for the individualization of non-small-cell lung cancer patients treatment. Oncotarget 2017;8:60291-8.

4. Provencio M, Torrente M, Calvo V, Pérez-Callejo D, Gutiérrez L, Franco $\mathrm{F}$, et al. Prognostic value of quantitative ctDNA levels in non small cell lung cancer patients. Oncotarget 2018;9:488-94.

5. Dawson S-J, Tsui DWY, Murtaza M, Biggs H, Rueda OM, Chin S-F, et al. Analysis of circulating tumor DNA to monitor metastatic breast cancer. N Engl J Med 2013;368:1199-209.

6. Bettegowda C, Sausen M, Leary RJ, Kinde I, Wang Y, Agrawal N, et al. Detection of circulating tumor DNA in early- and late-stage human malignancies. Sci Transl Med 2014;6:224ra24.

7. Wang Y, Springer S, Zhang M, McMahon KW, Kinde I, Dobbyn L, et al. Detection of tumor-derived DNA in cerebrospinal fluid of patients with primary tumors of the brain and spinal cord. Proc Natl Acad Sci U S A 2015;112:9704-9.

8. Villatoro S, Mayo-de-las-Casas C, Jordana-Ariza N, Viteri-Ramírez S, Garzón-Ibañez M, Moya-Horno I, et al. Prospective detection of mutations in cerebrospinal fluid, pleural effusion, and ascites of advanced cancer patients to guide treatment decisions. Mol Oncol 2019;13:2633-45.

9. De Mattos-Arruda L, Mayor R, Ng CKY, Weigelt B, Martínez-Ricarte F, Torrejon D, et al. Cerebrospinal fluid-derived circulating tumour DNA better represents the genomic alterations of brain tumours than plasma. Nat Commun 2015;6:8839.
10. Pérez-Barrios C, Nieto-Alcolado I, Torrente M, Jiménez-Sánchez C, Calvo V, Gutierrez-Sanz L, et al. Comparison of methods for circulating cell-free DNA isolation using blood from cancer patients: impact on biomarker testing. Transl Lung Cancer Res 2016;5:665-72.

11. ICH Topic Q 2 (R1) Validation of Analytical Procedures: Text and Methodology Step 5 NOTE FOR GUIDANCE ON VALIDATION OF ANALYTICAL PROCEDURES: TEXT AND METHODOLOGY (CPMP/ ICH/381/95) APPROVAL BY CPMP November 1994 DATE FOR COMING INTO OPERATION; 1995.

12. Romero A, Jantus-Lewintre E, García-Peláez B, Royuela A, Insa A, Cruz $P$, et al. Comprehensive cross-platform comparison of methods for non-invasive EGFR mutation testing: results of the RING observational trial. Mol Oncol 2021;15:43-56.

13. Provencio M, Pérez-Barrios C, Barquin M, Calvo V, Franco F, Sánchez E, et al. Next-generation sequencing for tumor mutation quantification using liquid biopsies. Clin Chem Lab Med 2020;58: 306-13.

14. Liu D, Lu Y, Hu Z, Wu N, Nie X, Xia Y, et al. Malignant pleural effusion supernatants are substitutes for metastatic pleural tumor tissues in egfr mutation test in patients with advanced lung adenocarcinoma. PLoS One 2014;9. https://doi.org/10.1371/ journal.pone.0089946.

15. Li Z, Zhang Y, Bao W, Jiang C. Insufficiency of peripheral blood as a substitute tissue for detecting EGFR mutations in lung cancer: a meta-analysis. Target Oncol 2014;9:381-8.

16. Douillard JY, Ostoros G, Cobo M, Ciuleanu T, Cole R, McWalter G, et al. Gefitinib treatment in EGFR mutated caucasian NSCLC: circulating-free tumor DNA as a surrogate for determination of EGFR status. J Thorac Oncol 2014;9:1345-53.

17. Jiang BY, Li YS, Guo WB, Zhang XC, Chen ZH, Su J, et al. Detection of driver and resistance mutations in leptomeningeal metastases of NSCLC by next-generation sequencing of cerebrospinal fluid circulating tumor cells. Clin Cancer Res 2017; 23:5480-8.

18. Martínez-Ricarte F, Mayor R, Martínez-Sáez E, Rubio-Pérez C, Pineda E, Cordero E, et al. Molecular diagnosis of diffuse gliomas through sequencing of cell-free circulating tumor DNA from cerebrospinal fluid. Clin Cancer Res 2018;24:2812-9.

19. Pan W, Gu W, Nagpal S, Gephart MH, Quake SR. Brain tumor mutations detected in cerebral spinal fluid. Clin Chem 2015;61: 514-22.

20. Boire A, Brandsma D, Brastianos PK, Le Rhun E, Ahluwalia M, Junck $L$, et al. Liquid biopsy in central nervous system metastases: a RANO review and proposals for clinical applications. Oxford: Oxford University Press; 2019, vol 21: 571-83 pp.

21. Lin J, Gu Y, Du R, Deng M, Lu Y, Ding Y. Detection of EGFR mutation in supernatant, cell pellets of pleural effusion and tumor tissues from non-small cell lung cancer patients by high resolution melting analysis and sequencing. Int J Clin Exp Pathol 2014;7: 8813-22.

22. Guo Z, Xie Z, Shi H, Du W, Peng L, Han W, et al. Malignant pleural effusion supernatant is an alternative liquid biopsy specimen for comprehensive mutational profiling. Thorac Cancer 2019;10: 823-31.

23. O'Leary B, Hrebien S, Beaney M, Fribbens C, Garcia-Murillas I, Jiang J, et al. Comparison of BEAMing and droplet digital PCR for circulating tumor DNA analysis. Clin Chem 2019;65:1405-13. 
24. Song Z, Wang W, Li M, Liu J, Zhang Y. Cytological-negative pleural effusion can be an alternative liquid biopsy media for detection of EGFR mutation in NSCLC patients. Lung Cancer 2019;136:23-9.

25. Hata A, Katakami N, Yoshioka H, Kaji R, Masago K, Fujita S, et al. Spatiotemporal T790M heterogeneity in individual patients with EGFR-mutant non-small-cell lung cancer after acquired resistance to EGFR-TKI. J Thorac Oncol 2015;10:1553-9.

26. Su J, Zhong W, Zhang X, Huang Y, Yan H, Yang J, et al. Molecular characteristics and clinical outcomes of EGFR exon 19 indel subtypes to EGFR TKIs in NSCLC patients. Oncotarget 2017;8: $111246-57$.

27. Zhang P, Wu X, Tang M, Nie X, Li L. Detection of EGFR gene mutation status from pleural effusions and other body fluid specimens in patients with lung adenocarcinoma. Thorac Cancer 2019;10:2218-24.

28. Oxnard GR, Hu Y, Mileham KF, Husain H, Costa DB, Tracy P, et al. Assessment of resistance mechanisms and clinical implications in patients with EGFR T790M-positive lung cancer and acquired resistance to osimertinib. JAMA Oncol 2018;4:1527-34.

29. Gainor JF, Dardaei L, Yoda S, Friboulet L, Leshchiner I, Katayama $R$, et al. Molecular mechanisms of resistance to first- and secondgeneration ALK inhibitors in ALK -rearranged lung cancer. Cancer Discov 2016;6:1118-33.

Supplementary Material: The online version of this article offers supplementary material (https://doi.org/10.1515/cclm-2020-1465). 